\title{
WMAP2006: Cosmological Parameters and Large-scale Structure of the Universe
}

\author{
S. Apunevych, B. Venhlovska, Yu. Kulinich, B. Novosyadlyj
}

November 24, 2018

\author{
Astronomical observatory of Ivan Franko National University of Lviv \\ Kyryla i Methodia, 8, 79005 Lviv Ukraine
}

\begin{abstract}
The parameters of cosmological model with cold dark matter and cosmological constant $(\Lambda \mathrm{CDM})$ have been determined on a basis of three-year cosmic microwave background observations by space mission WMAP, as well as the data on the large-scale structure of the Universe. The data cover scales from 1 up to $10000 \mathrm{Mpc}$. The best-fit values of $\Lambda \mathrm{CDM}$ model parameters were found by minimization of $\chi^{2}$ using the Levenberg-Markquardt approach $\left(\Omega_{\Lambda}=0.736 \pm 0.065, \Omega_{m}=0.238 \pm 0.080, \Omega_{b}=\right.$ $0.05 \pm 0.011, h=0.68 \pm 0.09, \sigma_{8}=0.73 \pm 0.08$ and $\left.n_{s}=0.96 \pm 0.015\right)$. It is shown that the $\Lambda$ CDM model with these values of the parameters agrees well with the angular power spectrum of cosmic microwave background and with power spectra of the density perturbations, estimated from spatial distributions of galaxies, rich galaxy clusters and from statistics of Ly ${ }_{\alpha}$ absorption lines in spectra of distant quasars as well. The accordance of modeled characteristics of the large-scale structure with observable ones was analyzed, and possible reasons of significant discrepancies between some of them were considered.
\end{abstract}

Keywords: CMB temperature fluctuations, cosmological parameters, large-scale structure of Universe

\section{Introduction}

In 2001, the spacecraft of WMAP (Wilkinson Microwave Anisotropy Probe) had been set into circumsolar orbit at Lagrange point $L_{2}$. It started full-sky measurements of temperature fluctuations of the cosmic microwave background $(\mathrm{CMB})$ with resolution of $\approx 13^{\prime}$. In 2003 the results were published of processing data accumulated during the first year of observations [4, 21, 36, 40. This event signalized the beginning of a new stage in cosmology, the epoch of the precision cosmology. It was for the first time when the power spectrum of the CMB temperature fluctuations was determined in the widest range of angular scales from $20^{\prime}$ to $180^{\circ}$, this is equivalent to the interval of spherical harmonics $2 \leq \ell \leq 1000$. The WMAP data have provided us with credible confirmation of acoustic peaks existence and possibility to determine their positions and amplitudes. The acoustic peaks had been discovered at first in the balloon-borne experiments like BOOMERANG [5, 27, MAXIMA [19, 24] and by ground-based interferometer DASI 17. This "peaked" structure of the spectrum was an ultimate argument for the adiabatic character of primordial perturbations of matter density and space-time metrics. From these perturbations the galaxies and large-scale structure of the Universe have been formed. The relations between acoustic peaks amplitudes and positions within angular power spectrum indicate that $\Lambda \mathrm{CDM}$ model with the scale-invariant spectrum of the primordial scalar density perturbations properly describes the observed Universe.

The bounds were set for the values of six main parameters of the model 36, 1] on the basis of the WMAP data complemented by data set on the large-scale structure of the Universe, expansion rate and dynamics, abundance of light elements: $\Omega_{\Lambda}=0.7-0.8, \Omega_{m}=0.23-0.31, \Omega_{b}=0.04-0.05, h=0.68-0.75$, $A_{s}=0.75-0.92, n_{s}=0.9-0.96$ (the exact values depend on the set of the observational data used). From these determinations the space curvature follows to be close to zero, i.e. $\Omega_{k}=0-0.04$. In addition to the main parameters the constraint on the optical depth to the last scattering surface $\tau$ also was found at a level of $\tau=0.23$. The optical depth is caused by reionization of intergalactic environment by the first stars. Also the upper bounds were determined for allowable values of two other parameters, namely the 
amplitude of tensor mode of perturbations $\left(A_{t}\right)$ and the mass density of neutrino in units of the critical $\left(\Omega_{\nu}\right)$, see [1, 2, 28, 36.

On March, 2006 the WMAP team had released the data of three-year observations of CMB temperature fluctuations [16, 20, 29, 35. These data differ from previous due to advanced techniques used for treatment of the measurements noise and foreground contamination. As a result the signal-to-noise ratio was greatly improved so that the errors for each point of the CMB power spectrum have decreased as much as twice and correspondingly the errors of the determination of the amplitudes and positions of acoustic peaks and dips (troughs) have decreased two times too. The range of the allowable values considerably narrowed for the $\Lambda \mathrm{CDM}$ model parameters determined on basis of this spectrum [35]. The anisotropy of the CMB polarization was measured. This polarization was caused by rescattering of the CMB photons by free electrons generated by ionization of protogalaxies medium with the first generation of stars. The WMAP have detected the signal of $E$-mode polarization at lower spherical harmonics $\ell \approx 2 \ldots 6, \ell(\ell+1) C_{\ell}^{E E}=0.086 \pm 0.029(\mu K)^{2}$. It means the complete reionization took place at $z \approx 8-12$ and the optical depth to the last scattering surface is $\tau=0.09 \pm 0.03$ [29].

In this paper we determine the main parameters of the $\Lambda \mathrm{CDM}$ model using the new data on the amplitudes and positions of acoustic peaks and characteristics the large-scale structure of the Universe. Also we shall investigate the concordance between various observational data and models.

\section{Power spectrum of CMB temperature fluctuations}

The angular power spectrum evaluated by processing the three-year observations of the CMB is plotted in Fig. 国. The whole spherical harmonics range $\ell=2-1000$ is binned into 39 bins (marked by horizontal bars), the value of the amplitude was determined for each bin by special techniques with use of the full-sky map. The sources of foreground contamination were eliminated during this processing, as well as traces of Galaxy plane (see [20, 16] for details on data processing). The errors include measurements noise, guiding errors, signal calibration errors and statistic error connected to unremovable sample incompleteness (cosmic variance). The solid line designates the power spectrum computed with use of CMBFast code 42 for $\Lambda \mathrm{CDM}$ model with parameters giving the best-fit to the observed spectrum, $\Omega_{\Lambda}=0.76, \Omega_{m}=0.24$, $\Omega_{b}=0.042, h=0.73, A_{s}=0.83, n_{s}=0.958$. The goodness of fit can be estimated by statistics of $\chi^{2}$, the sum of squared differences between observed and modelled amplitudes of the spectrum divided by the value of variance for a central point of each bin. Assuming no correlation between the values of power in adjacent bins we get $\chi^{2}=37.8$ for 33 degrees of freedom. Also for comparison we have plotted by dotted line the spectrum of $\Lambda \mathrm{CDM}$ model with values of parameters given as best-fit to the data of first-year WMAP observations and large-scale structure of the Universe [1, 2], $\chi^{2}=68.2$ for this spectrum.

The positions and amplitudes of acoustic peaks and dips are important features of the angular power spectrum. As one can see, using the data of 2006 we can reliably determine the positions and amplitudes of first and second peaks and dip between them. The position of the third peak can not be determined reliably from these data, since resolution of the WMAP telescope is $\approx 13^{\prime}$. Only involving the data of ground-based interferometric and baloon-borne experiments with better angular resolution makes possible to determine the position and amplitude of third acoustic peak. However, WMAP2006 data alone indicate on power increase beyond the second peak. The numerical values for the peaks and dips positions and amplitudes are listed in Table 1 based on three-year WMAP observations 20. Also their values from first-year data release are quoted therein for comparison 4 .

As one can see, the precision of amplitudes determination has grown almost two times. For the first (primary) peak it is better than $1 \%$, for the other elements (except for the third peak) it is better than $2 \%$. In order to estimate the position and amplitude of the third peak the observations data from CBI 33] and ACBAR 23] experiments have been used. The CMB temperature fluctuations were measured in these experiments with high angular resolution over the separated areas on the sky. However, it could not be excluded that given precision of the third peak position and amplitude determination at the level of $\approx 15 \%$ does not take into account properly the systematic errors, as it follows from comparison the curve in Fig. 1 with data on the peak position from Table. 1 1

The Table 2 lists the values of $(\Delta T)_{\ell}^{2}=\ell(\ell+1) C_{\ell} / 2 \pi$ for the range of spherical harmonics $2 \leq \ell \leq 10$ (this segment belongs to the Sachs-Wolfe 'plateau'), as resulting from data of one-year and three-year observations. At these scales the main contribution to the uncertainty of amplitudes of real spectrum comes from cosmic variance, which is unavoidable because of incomplete statistics of the large areas on the sky (at spherical harmonics $\ell \leq 10$ ). However, this part of the spectrum is particularly valuable for

\footnotetext{
${ }^{1}$ the observation data, tables and maps, are available at http://lambda.gsfc.nasa.gov/
} 


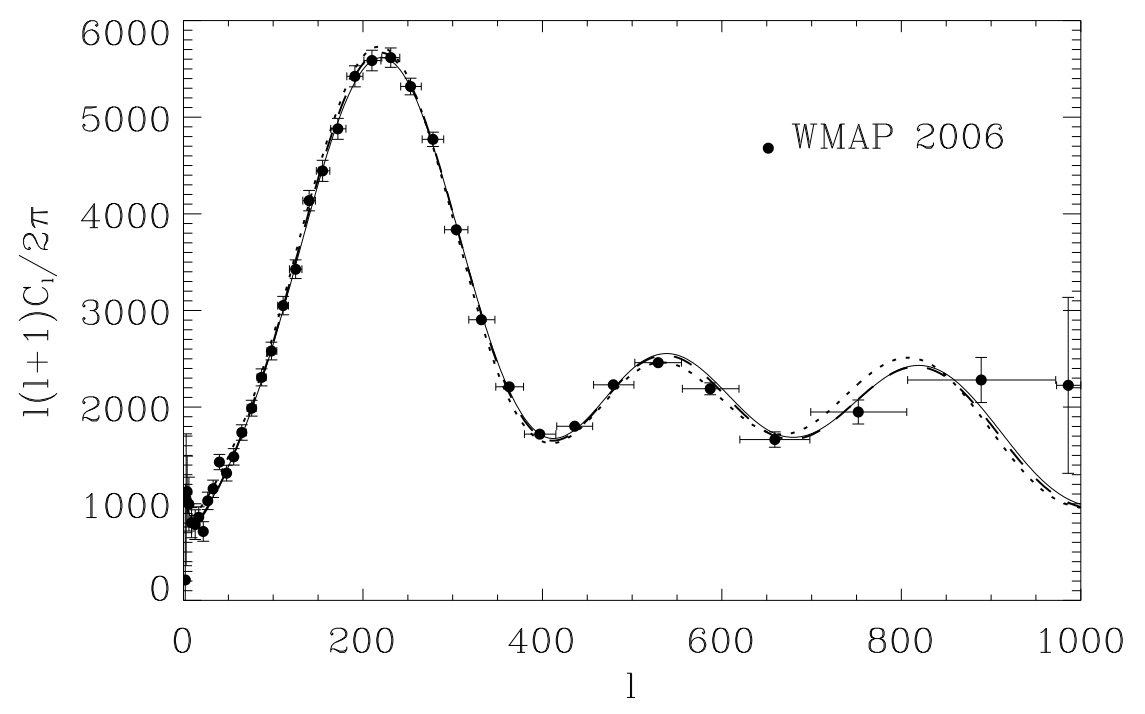

Figure 1: Angular power spectrum of CMB temperature fluctuations evaluated according the WMAP experiment data of three years of continuous observations. Solid line denotes the spectrum in best-fit $\Lambda \mathrm{CDM}$ model according to the data of [35] $\left(\chi^{2}=37.8\right)$. The dotted line denotes the spectrum for $\Lambda \mathrm{CDM}$ model which is the best-fit to 1-year WMAP data and large-scale structure observables [1, 2] $\left(\chi^{2}=68.2\right)$. The dashed line depicts the spectrum of $\Lambda$ CDM3 model with parameters found further in this paper $\left(\chi^{2}=37.2\right)$.

Table 1: The positions and amplitudes of acoustic peaks and dips in the CMB power spectrum as estimated by first-year and three-year WMAP observations.

\begin{tabular}{|c|c|c|c|c|}
\hline & & WMAP2003 & & WMAP2006 \\
\hline Name & $\begin{array}{c}\text { Position } \\
l\end{array}$ & $\begin{array}{c}\text { Amplitude } \\
(\Delta T)^{2}(\mu K)^{2}\end{array}$ & $\begin{array}{c}\text { Position } \\
l\end{array}$ & $\begin{array}{c}\text { Amplitude } \\
(\Delta T)^{2}(\mu K)^{2}\end{array}$ \\
\hline 1st peak & $220.1 \pm 0.8$ & $5583 \pm 73$ & $220.7 \pm 0.7$ & $5619 \pm 30$ \\
1st deep & $411.0 \pm 3.5$ & $1681 \pm 41$ & $412.8 \pm 1.9$ & $1704 \pm 27$ \\
2nd peak & $546.0 \pm 10.0$ & $2381 \pm 83$ & $531.3 \pm 3.5$ & $2476 \pm 40$ \\
2nd deep & & $674.6 \pm 12.1$ & $1668 \pm 85$ \\
3rd peak & $820.0 \pm 18.0^{*)}$ & $2150 \pm 860^{*)}$ & $1143 \pm 167^{* *)}$ & $2442 \pm 355^{* *)}$ \\
& & & & \\
\hline
\end{tabular}

*) + BOOMERANG + MAXIMA+DASI, ${ }^{* *)}+\mathrm{CBI}+\mathrm{ACBAR}$ 
Table 2: Amplitudes of the CMB power spectrum at low harmonics according to the data of 1st and 3 -years of the WMAP observations.

\begin{tabular}{|c|c|c|}
\hline & WMAP2003 & WMAP2006 \\
\hline$\ell$ & $(\Delta T)_{\ell}^{2}(\mu K)^{2}$ & $(\Delta T)_{\ell}^{2}(\mu K)^{2}$ \\
\hline & & \\
2 & $123 \pm 763$ & $211 \pm 860$ \\
3 & $612 \pm 608$ & $1041 \pm 664$ \\
4 & $757 \pm 504$ & $731 \pm 537$ \\
5 & $1257 \pm 432$ & $1521 \pm 453$ \\
6 & $696 \pm 380$ & $661 \pm 395$ \\
7 & $830 \pm 342$ & $1331 \pm 353$ \\
8 & $628 \pm 314$ & $671 \pm 322$ \\
9 & $815 \pm 292$ & $631 \pm 298$ \\
10 & $618 \pm 276$ & $751 \pm 280$ \\
& & \\
\hline
\end{tabular}

analysis since the information on the primordial space-time metrics perturbations is available precisely therein, and it is not distorted by any later effects of the spectrum shape changes.

We use these data to determine the main parameters of the $\Lambda$ CDM model complementing them by the data on amplitudes of the spectrum at low harmonics $\ell=2 \ldots 10$, as listed in Table 7 in the paper [20].

\section{WMAP2006: Cosmological parameters}

The analysis of full set of observations yields indications that the simplest variant of cosmological model capable to reconcile a heterogeneous information is the $\Lambda$ CDM model. It has main parameters, such as the Hubble parameter $H_{0}$ (or its dimensionless counterpart $h \equiv H_{0} / 100 \mathrm{~km} / \mathrm{s} / \mathrm{Mpc}$ ), the value of the cosmological constant $\Lambda$ (conventionally presented in the units of critical density, $\Omega_{\Lambda}=\Lambda / 3 H_{0}^{2}$ ), baryon matter density in units of critical density $\Omega_{b}$, matter density (baryon+cold dark matter) $\Omega_{m}$, the amplitude of primordial spectrum of scalar perturbations and its tilt $n_{s}$. The primordial spectrum $P_{s}(k)=A_{s} k^{n_{s}}$ is Fourier transformation of the two-point spatial correlation function of the matter fluctuations. Quite commonly instead of the power spectrum amplitude $A_{s}$ the more illustrative quantity $\sigma_{8}$ is used, the r.m.s amplitude of matter perturbations at the rich cluster scale $R=8 h^{-1} \mathrm{Mpc}$,

$$
\sigma_{8}^{2}=A_{s} \int_{0}^{\infty} T(k)^{2} k^{n_{s}+2} W^{2}(8 k) d k /(2 \pi)^{3 / 2},
$$

where $T(k)$ is transfer function dependent on main parameters of the model, $W(8 k)$ is Fourier transformation of the window function selecting out the volume with the mass of the rich galaxy cluster.

Another important parameter is an optical depth to the last scattering surface $\tau$ originated by the secondary reionization of baryon matter by the first luminous objects, massive stars and quasars. The $\Lambda \mathrm{CDM}$ model can be extended to include the tensor mode of perturbations with the primordial spectrum $P_{t}(k)=A_{t} k^{n_{t}}$ and contribution of hot dark matter consisting of $N_{\nu}$ species of neutrinos with non-zero rest mass, $\Omega_{\nu}=\Sigma m_{\nu} / 93.104 h^{2}$. Thus, the total number of independent parameters of cosmological model can reach 11. Nevertheless not all of them are really independent, for example the parameter of space curvature is determined by the matter content and the cosmological constant value: $\Omega_{k}=1-\Omega_{m}-\Omega_{\Lambda}$. The upper constraints for $A_{t}$ and $\Omega_{\nu}$ have been determined in a number of recent papers on determination of the cosmological parameters. The best-fit values for them appeared so close to zero that we can neglect these parameters when predicting the properties of large-scale structure of the Universe. So that we concentrate efforts on determination of main parameters of the $\Lambda$ CDM model, namely the $\Omega_{\Lambda}, \Omega_{m}, \Omega_{b}$, $h, A_{s}, n_{s}$. We have fixed the value of the optical depth at $\tau=0.09$ according to the results of the determination based on the CMB polarization fluctuations detected in the WMAP experiment [29].

The determination of the $\Lambda$ CDM parameters proceeds in the following steps. Let us have $N$ measured values of the characteristics of the large-scale structure, and the values of $n$ cosmological parameters 
must be found on this basis. We assume the probability distribution function for perturbations to be Gaussian and consider all observational data as mutually independent. The number of degrees of freedom for such system is $\nu=N-n$. The cosmological parameters are found by means of non-linear LevenbergMarkquardt minimization applied to the value of

$$
\chi^{2}=\sum_{j=1}^{N}\left(\frac{\tilde{y}_{j}-y_{j}}{\Delta \tilde{y}_{j}}\right)^{2},
$$

here $\tilde{y}_{j}$ is measured value of some $j$-th characteristics of large-scale structure, $y_{j}$ is corresponding theoretical prediction, $\Delta \tilde{y}_{j}$ is statistical error of the measured value, and $N$ is a total number of the observational characteristics. The accuracy of the model parameters determination depends not only on the precision of the measurements but on the accuracy of theoretical predictions $y_{j}$ calculation as well. One can use the code CMBFast [37, 42] or its modifications CAMBCODE [8, CMBEasy [1] to compute the power spectrum of the CMB temperature fluctuations and the power spectrum of the matter density perturbations. The system of the linear Einstein-Boltzmann equations for perturbations in multi-component medium is numerically solved in these codes. They provide inner accuracy up to $1 \%$ or better when evaluating CMB temperature fluctuations and the matter power spectra. The time required for the code to compute the single model is quite short, however its direct application in optimization problems requires significant computational resources since the large number of the models need to be calculated during minimum search. As alternative the semi-analytical methods or interpolations over the beforehand calculated grid can be used to evaluate the corresponding predictions. Precisely these methods are used in this paper. The accuracy is controlled by CMBfast code.

We evaluated peaks positions and amplitudes for predefined set of cosmological parameters in the following way. First, we complemented the CMBFast code by subroutine of searching extrema within domain the CMB power spectrum containing acoustic peaks. The extrema are output to file along with the corresponding values of parameters $\Omega_{\Lambda}, \Omega_{m}, h, \Omega_{b}, n_{s}$. At the next stage we construct the grid of values for amplitudes and positions of acoustic peaks and dips covering the region of parametric space $\Omega_{\Lambda}=0.0-0.8, \Omega_{m}=0.2-0.8, h=0.3-0.9$ (with step equal 0.1 ) and $\Omega_{b}=0.02-0.08, n_{s}=0.9-1.1$ (with step equal 0.01), for three values of curvature $\Omega_{k}=-0.05 ; 0.0 ; 0.05$. In every grid node the $A_{s}$ was determined by normalization of $\Delta T / T$ power spectrum according to 6 , with later renormalization within optimization algorithm. Note, that the renormalization coefficient never get out of the $0.9-1.1$ range. The acoustic peaks/dips positions and amplitudes for the values of parameters lying between the grid nodes were found by interpolation using 5-dimensional surface of second order. The comparison of directly calculated by CMBFast results has shown that the deviations between interpolated and precise values did not exceed $0.5 \%$.

The amplitude of the CMB temperature fluctuations power spectrum at low spherical harmonics was calculated using the semi-analytical techniques from [13], the accuracy was quite satisfactory for this region.

Besides the WMAP data we also used the data form other cosmological observations in cosmological parameters determination, namely the constraints on the Hubble constant $h=0.72 \pm 0.08$ [15], on baryon content $\Omega_{b} h^{2}=0.0214 \pm 0.002\left[22\right.$ and content of dark matter $\Omega_{m}-0.75 \Omega_{\Lambda}=-0.25 \pm 0.12531$, (these datasets are denoted "h", "BBN" and "SNIa" accordingly). Also data on of spatial distribution of galaxies and clusters, peculiar velocities, mass and X-ray temperature function of rich galaxy clusters, $L y_{\alpha}$-clouds in the intergalactic space were included ((LSS dataset). The list of observables consists of 112 experimental quantities with $1 \sigma$ errors (see Tabl. 4 and Fig. 36 6). We consider all measurements as statistically independent also assume that the probability distribution function of experimental errors obeys to the normal law. The detailed description of the procedure used for calculating the predictions of large-scale structure characteristics for the given initial power spectrum of density perturbations is given in our papers [12, 13. The transfer function $T(k)$ of the initial power spectrum of density perturbations in $\Lambda$ CDM model was computed with analytical approximation from [14].

The results of determination of the six main parameters of the $\Lambda$ CDM model are presented in Table 3 for three observational data sets: WMAP2006 data alone, data set WMAP2006+BBN+h+SNIa, data set WMAP2006+BBN+h+SNIa+LSS. These models are denoted as $\Lambda$ CDM1, $\Lambda$ CDM2 and $\Lambda$ CDM3 correspondingly. For last we also computed $95.4 \%$ confidence intervals of the values of every parameter $p_{i}(i=1,2, \ldots, 6)$ by integrating corresponding likelihood function $L\left(p_{i}\right)=\exp \left[-0.5 \Delta \chi^{2}\left(p_{i}\right)\right]$ (see [1, 2] for details). As it was in paper [1] the likelihood functions of parameters are symmetric with regard to the best-fit values and the Gaussian function is a good approximation for it. Thus the values of the parameters listed in the last column of Table 3 lie in the middle of corresponding ranges: $\Omega_{\Lambda}=0.67-0.80$, $\Omega_{m}=0.20-0.36, \Omega_{b}=0.04-0.06, h=0.59-0.76, n_{s}=0.945-0.975, \sigma_{8}=0.65-0.81$. The values for the 
Table 3: The best-fit values of parameters of $\Lambda$ CDM model according to different determinations

\begin{tabular}{|c|c|c|c|c|}
\hline Parameters & $\begin{array}{c}\text { Range of the best-fit } \\
\text { values from [35] }\end{array}$ & WMAP2006 & $\begin{array}{c}\text { WMAP2006+ } \\
\text { BBN+h+SNIa } \\
\Lambda \text { CDM2 }\end{array}$ & $\begin{array}{c}\text { WMAP2006+ } \\
\text { BBN+h+SNIa+LSS } \\
\Lambda \text { CDM3 }\end{array}$ \\
\hline$\Omega_{k}$ & $-(0.003-0.04)$ & -0.003 & -0.004 & -0.014 \\
$\Omega_{\Lambda}$ & $0.65-0.76$ & 0.771 & 0.763 & 0.736 \\
$\Omega_{m}$ & $0.23-0.30$ & 0.232 & 0.241 & 0.278 \\
$\Omega_{b}$ & $0.04-0.05$ & 0.040 & 0.041 & 0.050 \\
$h$ & $0.68-0.79$ & 0.76 & 0.74 & 0.68 \\
$n_{s}$ & $0.9-0.99$ & 0.97 & 0.97 & 0.96 \\
$\sigma_{8}$ & $0.7-0.83$ & 0.73 & 0.74 & 0.73 \\
\hline$\chi^{2} / \nu$ & & & & 0.98 \\
\hline
\end{tabular}

$\Lambda \mathrm{CDM} 1$ and $\Lambda$ CDM2 model parameters fit into this ranges too. As one can see, the best-fit values of parameters obtained with the use of the WMAP data alone coincide in practice with the results of the WMAP team determination (Tables 2 and 5 in 35]). It is an important achievement for cosmology that the WMAP2006-based determination of $h, \Omega_{b} h^{2}$ and $\Omega_{m}$ parameters agrees with independent 'direct' determinations by other authors [15, 22, 31. This gives the ground the $\Lambda$ CDM model with parameters as estimated here or in 35 to be called the 'concordance model' as it was proposed by [38. That is the reason why the inclusion of direct measurements of parameters to the input data reduces the value of $\chi^{2} / \nu$ relation.

The inclusion of data on the large-scale structure decreases the best-fit value for the Hubble constant to the 0.68 , but the values of other parameters have remained within the limits of the standard deviations $\sigma$ given by the WMAP team (Tables 2 and 5 in 35$]$ ). We should also point out on the 'stability' of the curvature parameter sign although its value is small and depends on the data set used in parameters determination. It could indicate on small but positive space curvature of observed Universe, so the hyper-surface of constant time should be finite in volume and ever expanding with increasing rate.

\section{WMAP2006: Large-scale structure}

Let us compute with CMBfast code the angular power spectrum of CMB temperature fluctuations, $\ell(\ell+1) C_{\ell} / 2 \pi$ for the $\Lambda$ CDM model with parameters listed in Table 3 in order to assess its accordance with observations. The spectrum is normalized by minimization of $\chi^{2}$ with summation over all points. The computation results are presented in Fig. 2, $\chi^{2}=54.6$ for the spectrum of $\Lambda$ CDM1 model, $\chi^{2}=53.3$ for $\Lambda$ CDM2 and $\chi^{2}=37.2$ for $\Lambda$ CDM3. Thus, the spectrum of $\Lambda$ CDM3 model fits the observational points of the spectrum with the same $\chi^{2}$ like in 35] (in Fig. 1 fits are overlayed). The Table 4 lists the observed characteristics of large-scale structure of the Universe, $\tilde{y}_{j} \pm \Delta \tilde{y}_{j}$, they were used within procedure of determination parameters for the $\Lambda$ CDM model. Their values in $\Lambda$ CDM model with parameters which correspond to the minimum of the $\chi^{2}$ by different determinations are quoted therein. The relative deviations of model values from observational, $\left(y_{j}-\tilde{y}_{j}\right) / \Delta \tilde{y}_{j}$, are given in parenthesis. The positive sign means that the modelled quantity is greater than observed one, negative sign means it is less. An asterisk marks quantities which were not used in procedure of parameters determination. As we can see, the most of characteristics of large-scale structure of the Universe as evaluated on the basis of $\Lambda$ CDM model spectra fall within the $1 \sigma$ range around the observational values and none of them fall outside of $3 \sigma$. Statistics of deviations complies to the normal distribution. Those LSS characteristics, for which observational values deviate from modelled for the $1 \sigma$ or more need more detailed analysis.

Now let us compare the power spectrum of matter density perturbations in the $\Lambda$ CDM 3 model with its estimates. The estimates were obtained by analysis of inhomogeneities found in the spatial distribution of galaxies and rich galaxy clusters. None of these estimates was used in procedure of determination of parameters. The spectrum is presented as dimensionless quantity $\Delta^{2}(k) \equiv P(k) k^{3} / 2 \pi^{2}$. It approximately equals to r.m.s. of matter density perturbations averaged over region with size of $\pi / k \mathrm{Mpc}$. In a such way we have built an independent criterion to estimate the validity of parameters of the given model. In Fig. 3 


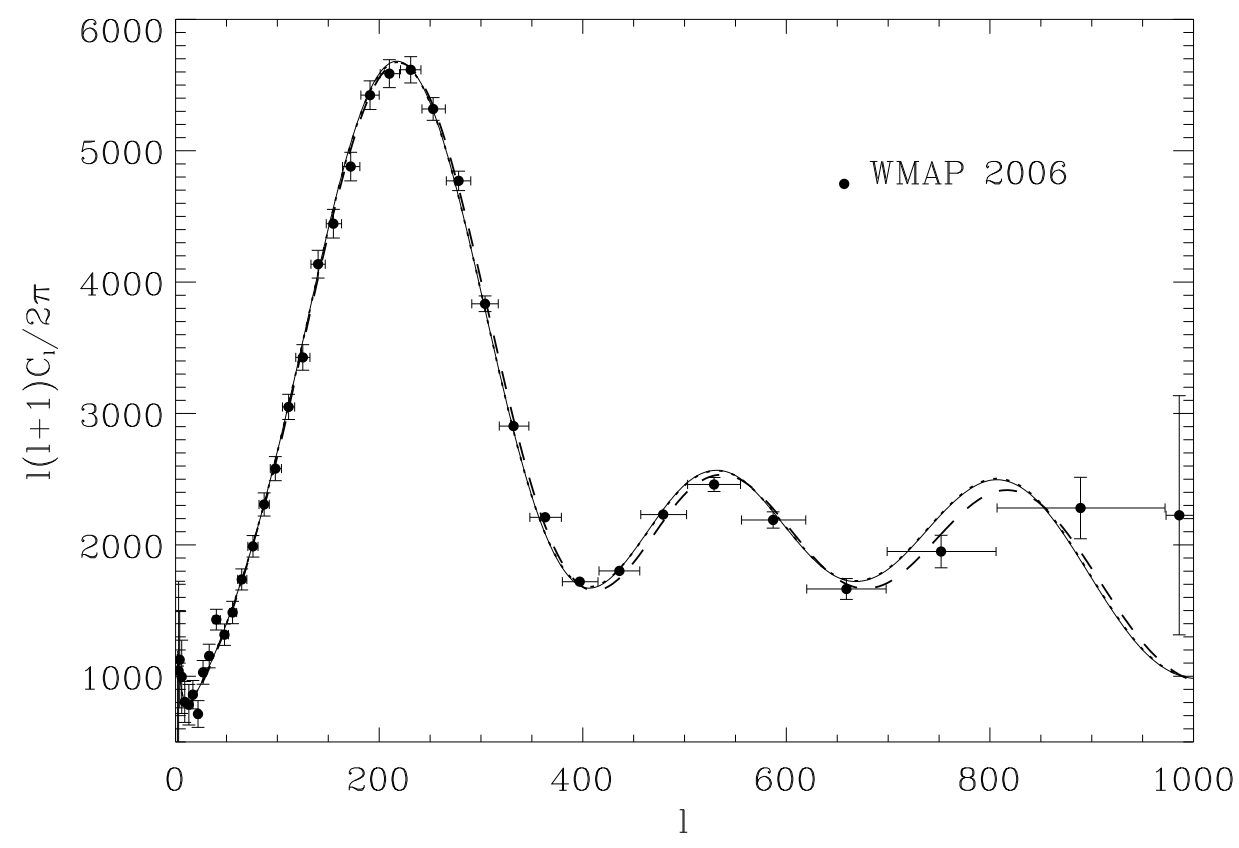

Figure 2: Power spectra of temperature fluctuations of CMB radiation in $\Lambda$ CDM models with parameters as in Table 3 (solid line for $\Lambda$ CDM1, dash-dotted line for $\Lambda$ CDM2, dashed for $\Lambda$ CDM3), points denote the observed by WMAP2006 spectrum.

two power spectra are plotted, one is estimated from spatial correlation function of rich galaxy clusters using the Abell and ACO catalogs 34 and the second from statistics of $\mathrm{Ly}_{\alpha}$ absorption lines in the spectra of distant quasars, the lines are originated by clouds of neutral hydrogen in intergalactic environment 9 . The experimental points of the second spectrum virtually "stack on" the modelled spectrum in the part of small scales $(k>1 h)$. The oscillations seen within the range of scales $0.2 h \leq k \leq 0.8 h$ come from of spatial limitations on sampling so they do not represent the real behavior of the perturbation spectrum. In the paper [9] the spectrum is given for $z=2.72$, so we recalculated its amplitude to the $z=0$ using the evolution law for perturbations from linear theory

$$
P(k)=P(k ; z) D_{1}^{2}(0) / D_{1}^{2}(z)
$$

where $P(k ; z)$ is the power spectrum for arbitrary $z, D_{1}(z)$ is a linear factor of growth which is well approximated by an analytical formula [7]

$$
D_{1}(z)=\frac{5}{2} \frac{\Omega(z)}{1+z}\left[\frac{1}{70}+\frac{209 \Omega(z)-\Omega^{2}(z)}{140}+\Omega^{4 / 7}(z)\right]^{-1},
$$

$\Omega(z)=\Omega_{m}(1+z)^{3} /\left(\Omega_{m}(1+z)^{3}+\Omega_{\Lambda}\right)$. Such concordance of the power spectrum of matter density perturbations in the $\Lambda$ CDM3 model with the same spectrum evaluated on the basis of the observed data in [9] is a good reason for model plausibility.

Since the bright galaxies and rich galaxy clusters were formed in highest peaks of matter density perturbations the power spectrum of inhomogeneous distribution of these objects relates to the spectrum of matter density through the biasing parameter $b: P(k)_{g, c l}=b_{g, c l}^{2} P(k)$ [3]. We determined $b$ for the spectrum 34] by minimization of deviations $b^{2} \Delta^{2}(k)$ from $\Delta_{A+A C O}^{2}\left(k_{I}\right)$ using the Levenberg-Markquardt method: $b_{A+A C O}=2.86$. As one can see from Fig. 3 the shape of spectrum $P_{A+A C O}(k) / b_{A+A C O}^{2}$ is well approximated by the spectrum of the $\Lambda$ CDM3 model. With increase of scale ( $k$ decreases) in the range $k \leq 0.05 h \Delta_{A+A C O}^{2}$ falls down steeper than $\Delta_{\Lambda C D M}^{2}$. The cause is that the sample of rich galaxy clusters used in [34] is spatially limited $\left(\leq 300 h^{-1} \mathrm{Mpc}\right)$, a similar spectrum from sample with 1 Gpc scale obtained in 26] and presented in Fig 4 confirms that. The scale-independent biasing parameter $b_{c l}=3.92$ therein was determined in the same way as in previous case. The apparent rise of the spectrum on large scales most probably is caused by dependence of bias upon a scale, that was not taken into account. Indeed, the 
Table 4: The characteristics of large-scale structure of Universe: the observables along with their values for $\Lambda \mathrm{CDM}$ models with parameters corresponding to the minimum of $\chi^{2}$ by different determinations. The deviations are quoted in parentheses for modelled quantities and observables in units of $1 \sigma$ error.

\begin{tabular}{|c|c|c|c|c|}
\hline Quantity & Observable & WMAP2006 & $\begin{array}{l}\text { WMAP2006+ } \\
\text { BBN+h+SNIa }\end{array}$ & $\begin{array}{c}\text { WMAP2006+ } \\
\text { BBN+h+SNIa+LSS }\end{array}$ \\
\hline$l_{p_{1}}$ & $220.7 \pm 0.720$ & $220.3(-0.54)$ & $220.4(-0.43)$ & $219.8(-1.29)$ \\
\hline$(\Delta T)_{p_{1}}^{2},(\mu K)^{2}$ & $5619 \pm 3020$ & $5619(0.00)$ & $5615(-0.13)$ & $5604(-0.51)$ \\
\hline$l_{d_{1}}$ & $412.8 \pm 1.9[20$ & $412.3(-0.26)$ & $412.0(-0.42)$ & $412.3(-0.39)$ \\
\hline$(\Delta T)_{d_{1}}^{2},(\mu K)^{2}$ & $1704 \pm 27[20$ & $1668(-1.35)$ & $1670(-1.28)$ & $1673(-1.15)$ \\
\hline$l_{p_{2}}$ & $531.3 \pm 3.5[20$ & $536.3(+1.42)$ & $536.2(+1.39)$ & $537.4(+1.75)$ \\
\hline$(\Delta T)_{p_{2}}^{2},(\mu K)^{2}$ & $2476 \pm 40[20]$ & $2534(+1.44)$ & $2539(+1.57)$ & $2558(+2.05)$ \\
\hline $\bar{l}_{d_{2}}$ & $674.6 \pm 12.1[20]$ & $674.4(-0.02)$ & $674.1(-0.04)$ & $679.7(+0.42)$ \\
\hline$(\Delta T)_{d_{2}}^{2},(\mu K)^{2}$ & $1668 \pm 85[20$ & $1692(+0.28)$ & $1700(+0.38)$ & $1687(+0.22)$ \\
\hline$l_{p_{3}}$ & $1143 \pm 167[20$ & *) 814.5 (-1.97) & *) $814.3(-1.97)$ & *) 820.2 (-1.93) \\
\hline$(\Delta T)_{p_{3}}^{2},(\mu K)^{2}$ & $2442 \pm 35520$ & *) $2451(+0.03)$ & *) $2463(+0.06)$ & *) $2439(-0.01)$ \\
\hline$(\Delta T)_{2}^{2},(\mu K)^{2}$ & $211 \pm 860[20$ & $993(+0.91)$ & $980(+0.89)$ & $1013(+0.93)$ \\
\hline$(\Delta T)_{3}^{2},(\mu K)^{2}$ & $1041 \pm 664[20$ & $940(-0.15)$ & $934(-0.16)$ & $983.4(-0.93)$ \\
\hline$(\Delta T)_{4}^{2},(\mu K)^{2}$ & $731 \pm 537[20$ & $892(+0.30)$ & $889(+0.29)$ & $942.5(+0.39)$ \\
\hline$(\Delta T)_{5}^{2},(\mu K)^{2}$ & $1521 \pm 45320$ & $877(-1.42)$ & $873(-1.43)$ & $923.7(-1.32)$ \\
\hline$(\Delta T)_{6}^{2},(\mu K)^{2}$ & $661 \pm 395[20$ & $851(+0.48)$ & $849(+0.48)$ & $903.4(+0.61)$ \\
\hline$(\Delta T)_{7}^{2},(\mu K)^{2}$ & $1331 \pm 35320$ & $835(-1.41)$ & $835(-1.41)$ & $892.5(-1.24)$ \\
\hline$(\Delta T)_{8}^{2},(\mu K)^{2}$ & $671 \pm 322[20]$ & $826(+0.48)$ & $827(+0.48)$ & $887.6(+0.67)$ \\
\hline$(\Delta T)_{9}^{2},(\mu K)^{2}$ & $631 \pm 298[20]$ & $822(+0.64)$ & $824(+0.65)$ & $886.6(+0.86)$ \\
\hline$(\Delta T)_{10}^{2},(\mu K)^{2}$ & $751 \pm 280[20$ & $820(+0.25)$ & $843(+0.26)$ & $888.3(+0.49)$ \\
\hline$h$ & $0.72 \pm 0.08[15$ & ${ }^{*)} 0.755(+0.44)$ & $0.744(+0.30)$ & $0.676(-0.55)$ \\
\hline$\Omega_{b} h^{2}$ & $0.0214 \pm 0.002[22]$ & ${ }^{*)} 0.0228(+0.70)$ & $0.0228(+0.71)$ & $0.0228(+0.72)$ \\
\hline$\Omega_{m}-0.75 \Omega_{\Lambda}$ & $-0.25 \pm 0.12531$ & ${ }^{*)}-0.35(-0.80)$ & $-0.35(-0.77)$ & $-0.27(-0.19)$ \\
\hline$V_{50}, \mathrm{~km} / \mathrm{s}$ & $370 \pm 110$ & ${ }^{*)} 251(-1.08)$ & *) $255(-1.05)$ & $271(-0.90)$ \\
\hline$\Delta_{\rho}$ & $0.54 \pm 0.139$ & *) $0.57(+0.20)$ & *) $0.59(+0.38)$ & $0.55(+0.10)$ \\
\hline$n_{p}$ & $-2.47 \pm 0.06[9]$ & *) $-2.49(-0.37)$ & *) $-2.49(-0.35)$ & $-2.50(-0.55)$ \\
\hline$\Delta_{\rho}$ & $0.72 \pm 0.0925$ & ${ }^{*)} 0.57(-1.67)$ & *) $0.60(-1.33)$ & *) $0.56(-1.79)$ \\
\hline$n_{p}$ & $-2.55 \pm 0.10[25$ & *) $-2.53(+0.19)$ & *) $-2.53(+0.19)$ & *) $-2.54(+0.09)$ \\
\hline$\sigma_{8}$ & $0.56 \pm 0.07141$ & *) $0.36(-2.82)$ & ${ }^{*)} 0.38(-2.54)$ & $0.40(-2.26)$ \\
\hline$\sigma_{c l}$ & $0.508 \pm 0.02932$ & ${ }^{*)} 0.44(-2.34)$ & ${ }^{*)} 0.45(-2.00)$ & $0.46(-1.33)$ \\
\hline
\end{tabular}

*) Not used in search procedure.

brighter clusters get an advantage on large distances, they was formed in higher peaks of matter density perturbations so they look more clustered (see for example [39]).

At the next step we compare the model spectra with the spectra obtained on the basis of observed spatial distribution of galaxies. The treatment of galaxies power spectra at small scales $\left(k>0.3 h \mathrm{Mpc}^{-} 1\right)$ is complicated by nonlinear distortions, the scale-dependence of biasing and by correlation between the values of its amplitude in the adjacent wave-number ranges as well. Therefore we have restricted ourself to the section of galactic spectrum starting from $k \leq 0.3 h \mathrm{Mpc}^{-1}$ when doing comparison with linear spectrum of the $\Lambda \mathrm{CDM}$ model. Also we assume biasing to be independent on $k$ in this range of scales. The special techniques were proposed in [18] for decorrelating data points. It allows to decrease the correlations in the range of nonlinearity and virtually removes them in the linear range. The decorrelated power spectrum of the PSRCz survey made of IRAS galaxies (Point Source Redshift Catalog) is presented (see Fig. 5). We have determined the biasing parameter for this spectrum by $\chi^{2}$-minimization by the Levenberg-Markquardt method and it has appeared to be close to unity, $b_{P S R C z}=1.08$, the proper value for galaxies from IRAS survey. The same power spectrum for Sloan Digital Sky Survey (SDSS) is presented in Fig. 6. The biasing parameter is $b_{S D S S}=1.21$. The amplitudes of the galactic spectra were divided by squared corresponding biasing factors to make the comparison with linear power spectrum of $\Lambda \mathrm{CDM}$ model. The linear power spectrum of matter density perturbations of the $\Lambda$ CDM model 

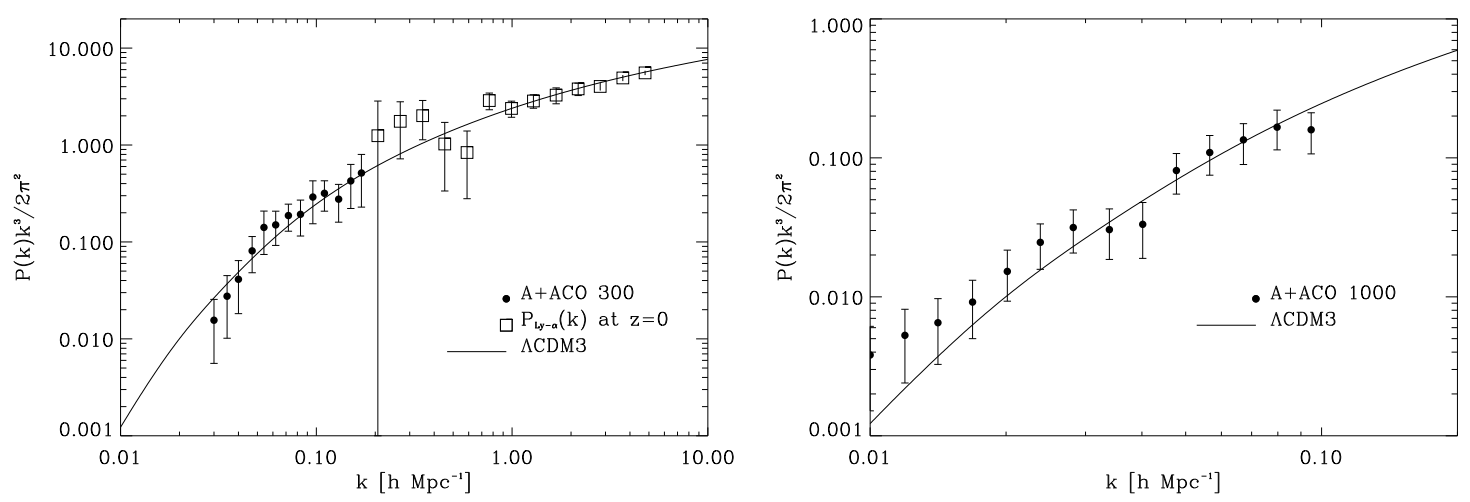

Figure 3: Power spectrum of matter density fluctuations as evaluated on the basis of spatial distribution of rich galaxy clusters from Abell-ACO catalog [34] $\left(P(k)=P_{A+A C O}(k) / b_{A+A C O}^{2}, b_{A+A C O}=2.86\right)$ as well as statistics of $\mathrm{Ly}_{\alpha}$ absorption lines in the spectra of distant quasars 9$]\left(P_{L y_{\alpha}}\right)$. The power spectrum of matter fluctuations in $\Lambda$ CDM3 model is plotted by solid line.

Figure 4: Power spectrum of matter density fluctuations as evaluated on the basis of spatial distribution of rich galaxy clusters from Abell-ACO catalog [26]: $P(k)=P_{A+A C O}(k) / b_{A+A C O}^{2}, b_{A+A C O}=3.92$. The power spectrum of matter fluctuations in $\Lambda$ CDM3 model is plotted by solid line.

conforms quite well to the amplitudes and shapes (the dependence on $k$ ) of the observed power spectra of inhomogeneities in the spatial distributions of galaxies.
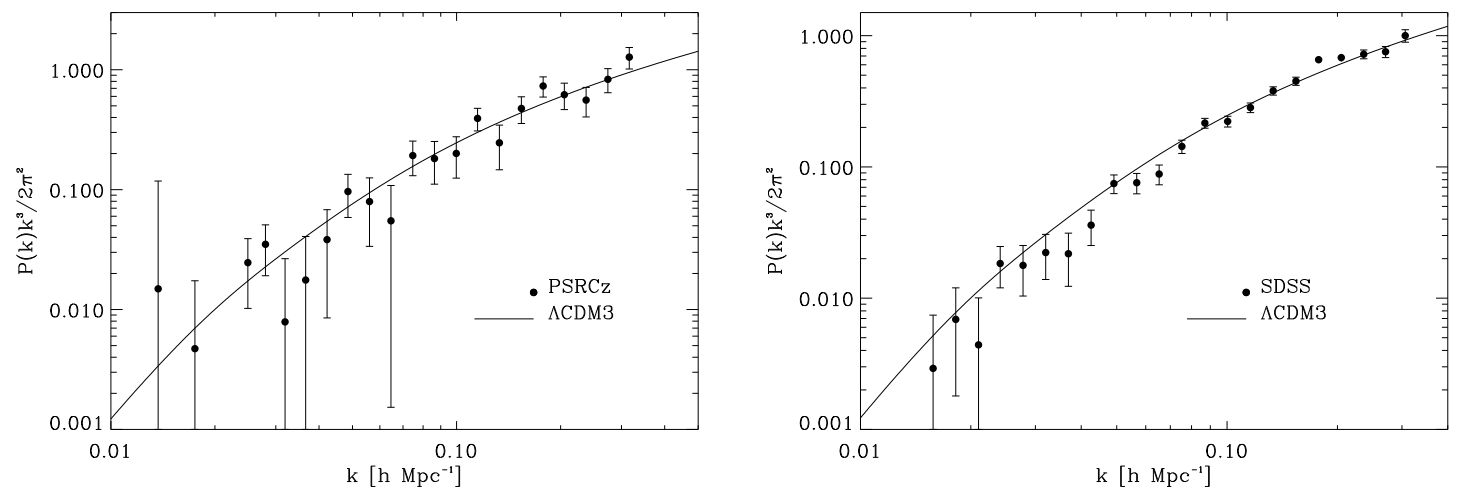

Figure 5: The power spectrum of matter density fluctuations as obtained on the basis of spatial distribution of IRAS galaxies in Point Source Catalog Redshift Survey (PSCRz) [18. P $(k)=P_{P S R C z}(k) / b_{P S R C z}^{2}$, $b_{P S R C z}=1.08$. The power spectrum of density fluctuations in $\Lambda$ CDM3 model is plotted by solid line.

Figure 6: The power spectrum of matter density fluctuations as obtained on the basis of spatial distribution of galaxies in Sloan Digital Sky Survey (SDSS) [39]. $P(k)=P_{S D S S}(k) / b_{S D S S}^{2}, b_{S D S S}=1.21$. The power spectrum of density fluctuations in $\Lambda$ CDM3 model is plotted by solid line.

\section{Discussion of results and conclusions}

Now we assess the concordance of two theoretical spectra with observed ones for two models the above mentioned $\Lambda$ CDM3 and $\Lambda$ CDM model from 35] with parameters $\Omega_{\Lambda}=0.76, \Omega_{m}=0.24, \Omega_{b}=0.042$, $h=0.73, A_{s}=0.83, n_{s}=0.958$. The values of $\chi^{2}$ for all these power spectra are listed in Table 5 . The values of biasing factors computed in the same way for both models are presented in parentheses for the power spectra from spatial distribution of galaxies and clusters. As it can be seen, the power spectrum of $\Lambda$ CDM3 model provides the fit to the most of observed spectra with smaller value of $\chi^{2}$ (except for 
$P_{A+A C O}(k)[26$ ) than $\Lambda \mathrm{CDM}$ model does 35 . Thus, among two models the $\Lambda$ CDM3 model could be deemed as closer to true model of Universe, which is still searched.

Table 5: $\chi^{2}$ for different spectra of $\Lambda$ CDM3 and $\Lambda$ CDM models [35.

\begin{tabular}{|c|c|c|}
\hline & $\chi^{2} \Lambda$ CDM[35] & $\chi^{2} \Lambda$ CDM3 \\
\hline$\ell(\ell+1) C_{\ell} / 2 \pi[20]$ & 37.8 & 37.2 \\
$P_{L y_{\alpha}}(k)[9]$ & 17.81 & 8.13 \\
$P_{A+A C O}(k)[34]$ & $4.22(b=2.70)$ & $4.02(b=2.86)$ \\
$P_{A+A C O}(k)[26$ & $8.44(b=3.70)$ & $9.43(b=3.92)$ \\
$P_{P S R C z}(k)[18]$ & $14.68(b=1.03)$ & $14.03(b=1.08)$ \\
$P_{S D S S}(k)[39]$ & $46.93(b=1.16)$ & $39.29(b=1.21)$ \\
\hline
\end{tabular}

Basing on confrontation of predicted for $\Lambda$ CDM model characteristics of LSS with observed ones as presented in Table 4 and Fig. 2 6 we should point out that regardless of pretty good concordance of the data set in a whole there are still "stable" deviations of some quantities beyond the $1 \sigma$ confidence limits. The deviation is called "stable" if it can not be removed by the change of set of the observational data. Namely, they are:

- the positions of 2 nd and 3rd acoustic peaks, $\ell_{p_{2}}, \ell_{p_{3}}$;

- amplitudes of 1st dip, 2nd and 3rd acoustic peaks, $(\Delta T)_{d_{1}}^{2},(\Delta T)_{p_{2}}^{2},(\Delta T)_{p_{3}}^{2}$;

- amplitudes of 5th and 7th spherical harmonics, $(\Delta T)_{5}^{2},(\Delta T)_{7}^{2}$;

- amplitude of the power spectrum of density perturbations based of X-ray temperature function $\left(\sigma_{8}\right.$ 41]) and mass function of rich galaxy clusters $\left(\sigma_{c l}\right.$ [32]).

The following explanations could be proposed for these deviations: i) excessive "stiffness" of $\Lambda \mathrm{CDM}$ model, ii) an assumption of scale-invariance of primordial perturbations spectrum, iii) the underestimation of $1 \sigma$ C.L. for some of experimental quantities. Obviously, the extension of the $\Lambda$ CDM models towards inclusion of dark energy or inflation models giving scale-dependent power spectrum demands for to the possibility of verification by high-quality observational data.

Hence, the data of observational cosmology spreading over scales from $1 \mathrm{Mpc}$ to $10000 \mathrm{Mpc}$ indicate that $\Lambda \mathrm{CDM}$ model with parameters $\Omega_{\Lambda}=0.736, \Omega_{m}=0.278, \Omega_{b}=0.05, h=0.68, \sigma_{8}=0.73$ and $n_{s}=0.96$ is the best-fit for whole data set. So this model can be considered as the closest to the true model of Universe within the class of 6-parameter cosmological models.

\section{References}

[1] Novosyadlyj B., Apunevych S. Cosmological Constraints on the amplitude of relic gravitational waves //Journal of Physical Studies.-2005.- 9.-P. 280-291. (in Ukrainian)

[2] Apunevych S., Novosyadlyj B. Constraints on relic gravitational waves from CMB and LSS observations // Proceeding of Gamov memorial conference "Astrophysics and cosmology after Gamov theory and observations" / Eds. A. Zhuk, G.S. Bisnovaty-Kogan, S. Silich and R. Terlevich. Cambridge Scientific Publishers Ltd. -2005.-P. 105-112.

[3] Bardeen J. M., Bond J. R., Kaiser N., Szalay A. S. The statistics of peaks of Gaussian random fields // Astrophys. J.-1986.-304.-P. 15-61.

[4] Bennett C. L., Halpern M., Hinshaw G., et al. First-year Wilkinson Microwave Anisotropy Probe (WMAP) observations: preliminary maps and basic results // Astrophys. J. Suppl. Ser.-2003.-148.P. 1-28.

[5] de Bernardis P., Ade P. A. R., Bock J. J., et al. A flat Universe from high-resolution maps of the cosmic microwave background radiation // Nature.-2000.-404.-P. 995-999.

[6] Bunn E. F., White M. The 4 year COBE normalization and large-scale structure // Astrophys. J.-1997.-480.-P. 6. 
[7] Carroll S. M., Press W. H., Turner E. L. The cosmological constant // Ann. Rev. Astron. and Astrophys.-1992.-30.-P. 499-542.

[8] Challinor A., Lewis A. Lensed CMB power spectra from all-sky correlation functions // Phys.Rev.2005.-D71-103010.

[9] Croft R. A. C., Hu W., Dave R. Cosmological limits on the neutrino mass from the Ly $\alpha$ forest // Phys. Rev. Lett.-1999.-83.-P. 1092.

[10] Dekel A., Eldar A., Kolatt T., et al. POTENT reconstruction from mark III velocities // Astrophys. J.-1999.-522.-P. 1-38.

[11] Doran M. CMBEASY: an Object Oriented Code for the Cosmic Microwave Background //JCAP.2005.-0510.-011.

[12] Durrer R., Novosyadlyj B. Cosmological parameters from complementary observations of the Universe // Mon. Notic. Roy. Astron. Soc.-2001.-324.-P. 560-572.

[13] Durrer R., Novosyadlyj B., Apunevych S. Acoustic peaks and dips in the cosmic microwave background power spectrum: observational data and cosmological constraints // Astophys. J.-2003.-583.P. 33-48.

[14] Eisenstein D. J., Hu W. Power spectra for cold dark matter and its variants // Astrophys. J.-1999.511.-P. 5-15.

[15] Freedman W. L., Madore B. F., Gibson B. K., et al. Final results from the Hubble Space Telescope Key Project to measure the Hubble constant // Astrophys. J.-2001.-553.-P. 47.

[16] Jarosik N., Barnes C., Greason M. R., et al. Three-year Wilkinson Microwave Anisotropy Probe (WMAP) observations: beam profiles, data processing, radiometer characterization and systematic error limits // Astrophys. J.-2006.- (astro-ph/0603452).

[17] Halverson N. W., Leitch E. M., Pryke C., et al. DASI first results: a measurement of the cosmic microwave background angular power spectrum // Astrophys. J.-2002.-568.-P. 38-45.

[18] Hamilton A. J. S., Tegmark M. The real-space power spectrum of the PSRCz survey from 0.01 to 300Mpc/h // Mon. Notic. Roy. Astron. Soc.-2002.-330.-P. 506-530.

[19] Hanany S., Ade P., Balbi A., et al. MAXIMA-1: a measurement of cosmic microwave background on angular scales of $10^{\prime}-5^{\circ} / /$ Astrophys. J.-2000.-545.-P. L5-L9.

[20] Hinshaw G., Nolta M. R., Bennett C. L., et al. Three-year Wilkinson Microwave Anisotropy Probe (WMAP) observations: temperature analysis // Astrophys. J.-2006.- (astro-ph/0603451).

[21] Hinshaw G., Spergel D. N., Verde L., et al. First-year Wilkinson Microwave Anisotropy Probe (WMAP) observations: the angular power spectrum // Astrophys. J. Suppl. Ser.-2003.-148.-P. 135159 .

[22] Kirkman D., Tytler D., Suzuki N., et al. The cosmological baryon density from the deuteriumto-hydrogen ratio in QSO absorption systems: D/H toward Q1243+3047 // Astrophys. J. Suppl. Ser.-2003.-149.-P. 1-28.

[23] Kuo C. L., et al. High-Resolution Observations of the Cosmic Microwave Background Power Spectrum with ACBAR // Astrophys. J. -2004.-600.-1.-P. 32-51.

[24] Lee A. T., Ade P., Balbi A., et al. High spatial resolution analysis of the MAXIMA-1 cosmic microwave background anisotropy data // Astrophys. J.-2001.-561.-P. L1-L5.

[25] McDonald P., Miralda-Escudé J., Rauch M., et al. The observed probability distribution function, power spectrum, and correlation function of the transmitted flux in the Ly $\alpha$ forest // Astrophys. J.-2000.-543.-P. 1-23.

[26] Miller C. J., Batuski D. J. The power spectrum of rich clusters on near-gigaparsec scales // Astrophys. J.-2001.-551.-P. 635-642. 
[27] Netterfield C. B., Ade P. A. R., Bock J. J., et al. A measurement by BOOMERANG of miltiple peaks in the angular power spectrum of the cosmic microwave background // Astrophys. J.-2002.-571. - P. $604-614$

[28] Novosyadlyj B., Apunevych S. The constraints on power spectrum of relic gravitational waves from current observations of large-scale structure of the Universe // Kinematics and physics of celestial bodies. Suppl.-2005-5.- P. 199-204.

[29] Page L., Hinshaw G., Komatsu E., et al. Three-year Wilkinson Microwave Anisotropy Probe (WMAP) observations: Polarization analysis // Astrophys. J.-2006.- (astro-ph/0603450).

[30] Press W. H., Teukolsky S. A., Vetterling W. T., Flannery B. P. Numerical recipes in FORTRAN.The art of scientific computing - New York:Cambridge Univ.Press, 1992.

[31] Perlmutter S., Aldering G., Goldhaber G., et al. Measurements of Omega and Lambda from 42 high-redshift supernovae // Astrophys. J.-1999.-517.-P. 565-586.

[32] Pierpaoli E., Scott D., White M. Power-spectrum normalization from the local abundance of rich clusters of galaxies // Mon. Notic. Roy. Astron. Soc.-2001.-325.-P. 77-88.

[33] Readhead A.C.S., et al. Extended mosaic observations with the Cosmic Background Imager // Astrophys. J..-2004.-609.-P. 498-512.

[34] Retzlaff J., Borgani S., Gottlober S., et al. Constraining cosmological models with cluster power spectra // New Astron. Rev.-1998.-3.-P. 631-646.

[35] Spergel D.N., Bean R., Dore O., et al. Three-year Wilkinson Microwave Anisotropy Probe (WMAP) observations: Implications for Cosmology // Astrophys. J.-2006.- (astro-ph/0603449).

[36] Spergel D. N., Verde L., Peiris H. V., et al. First-year Wilkinson Microwave Anisotropy Probe (WMAP) observations: determination of cosmological parameters // Astrophys. J. Suppl. Ser.-2003.148.-P. 175-215.

[37] Seljak U., Zaldarriaga M. A line-of-sight integration approach to cosmic microwave background anisotropies // Astrophys. J.-1996.-469.-P. 437-444.

[38] Tegmark M., Zaldarriaga M., Hamilton A. J. S. Towards a refined cosmic concordance model: joint 11-parameter constraints from CMB and large-scale structure // Phys. Rev. D.-2001.-63, N 4.

[39] Tegmark M., Blanton M. R., Strauss M. A., at al. The three-dimensional power spectrum of galaxies from the sloan digital sky survey // Astrophys. J.-2004.-606.-P. 702-740.

[40] Verde, L.; Peiris, H. V.; Spergel, D. N. et al. First-Year Wilkinson Microwave Anisotropy Probe (WMAP) Observations: Parameter Estimation Methodology // Astrophys. J. Suppl. Ser.-2003.-148.P.195-211.

[41] Viana P. T. P., Liddle A. W. Galaxy clusters at $0.3<z<0.4$ and the value of $\Omega_{0} / /$ Mon. Notic. Roy. Astron. Soc.-1999.-303.-P. 535-545.

[42] Zaldarriaga M., Seljak U. CMBFAST for spatially closed universes // Astrophys. J. Suppl. Ser.1999.-29, N 2.-P. 431-434. 